\title{
HENRI BERR
}

ET LA CULTURE DU XX $X^{\mathrm{e}}$ SIÈCLE 


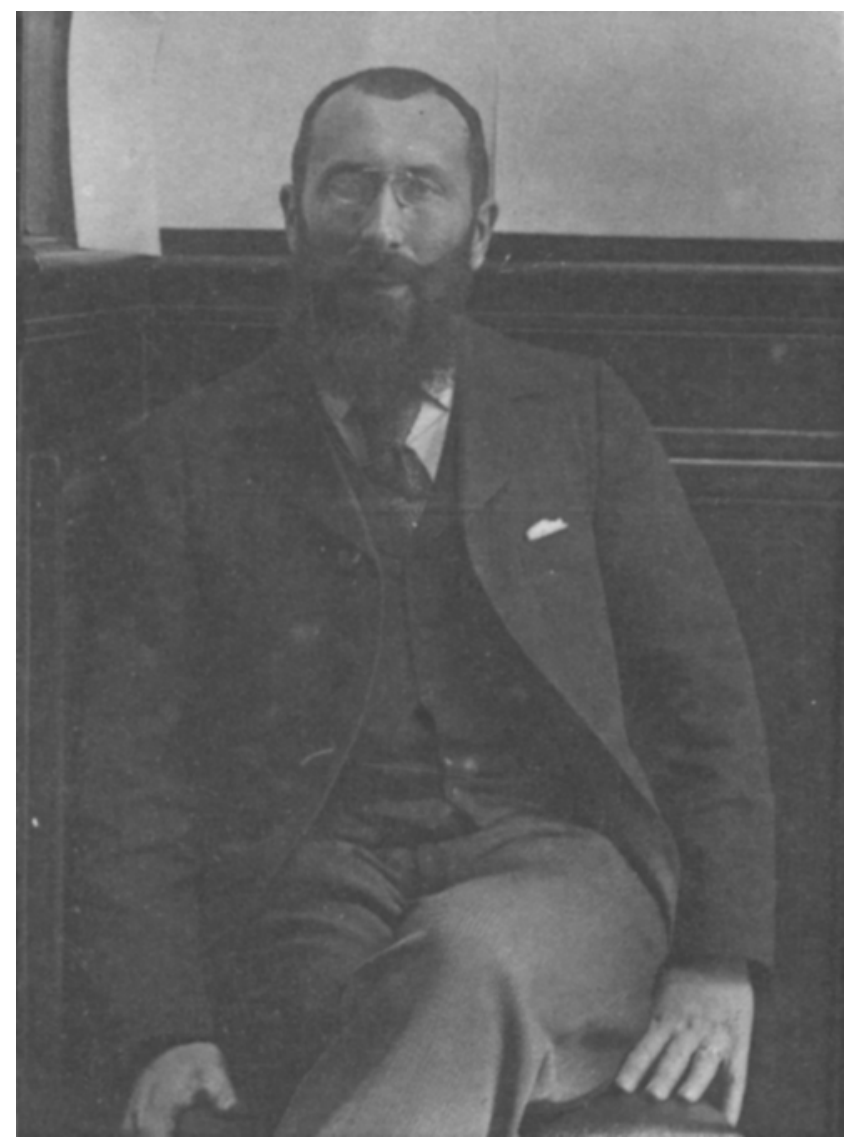




\title{
PRÉSENTATION
}

\section{HENRI BERR \\ (1863-1954)}

Dominique BoureL

\begin{abstract}
Alors que des parutions récentes ${ }^{1}$ documentent les derniers développements de la science historique française, les actes de ce colloque ${ }^{2}$ présentent la pensée et l'action d'une figure souvent évoquée mais finalement assez peu connue et qui a pourtant joué un rôle éminent dans la culture de notre pays.

Pour intéressante que soit la vie d'Henri Berr, elle est inséparable de son cuvre et de ses propres ouvrages dont on trouvera une bibliographie à la fin du volume. Sa revue, Revue de synthèse historique, puis Revue de synthèse, sa collection «L'Évolution de l'humanité », et enfin son Centre international de synthèse constituent une étape décisive dans l'histoire culturelle française, d'autant qu'ils sont toujours en activité aujourd'hui.

Fils d'un artisan des Vosges devenu fabricant de gants, il naît à Lunéville en 1863. D'une famille juive assimilée ${ }^{3}$ dont il parle assez peu mais qu'il fait lui-même remonter jusqu'au XVIII' siècle, il entre à l'ENS en 1881, avec une dispense d'âge après de brillantes études au lycée Charlemagne; il est de la même promotion que les philosophes Maurice Blondel et Frédéric Rauh. Revenant sur ses jeunes années, il écrit :
\end{abstract}

1. Pierre Nora (éd.), Essais d'ego-histoire, Paris, Gallimard, 1987. Pierre Chaunu, L'Instant éclaté, Entretiens avec François Dosse, Paris, Aubier, 1994. Giuliana Gemell, Fernand Braudel, Paris, Odile Jacob, 1995. Pierre DaIX, Braudel, Paris, Flammarion, 1995. Pierre VIDAL-NAQUET, Mémoires, t. I : La Brisure et l'attente, 1930-1955, Paris, Seuil-La Découverte, 1995. Pierre GouBERT, Un parcours d'historien. Souvenirs, 1915-1995, Paris, Fayard, 1996. Jacques LE GoFF, Une vie pour l'histoire. Entretiens avec Marc Heurgon, Paris, La Découverte, 1996. Gérard Norriel, Sur la "crise» de l'histoire, Paris, Belin, 1996.

2. «L'Histoire, la philosophie, la synthèse : Henri Berr (1863-1954) et la culture de son temps », 24-26 octobre 1994, à l'Institut Mémoire de l'édition contemporaine, Paris.

3. Françoise Јов, Les Juifs de Lunéville aux Xvire et XIXe siècles, Nancy, Presses universitaires de Nancy, 1989. 
«On me permettra ici un souvenir personnel qui donne à mon entreprise des racines profondes. À l'âge où l'on se cherche dans de naïs essais, littéraires ou scientifiques, vers la quinzième année, j'ai présomptueusement ébauché un "Discours sur l'Histoire universelle"... Avais-je lu le Discours de Bossuet? Je n'en suis pas sûr. Le titre, au moins, avait dû me frapper. Quoi qu'il en soit, j'ai eu de bonne heure le sentiment confus que l'histoire est le cœur même de la science. Et de plus en plus, il m'a semblé que la pensée a pour tâche essentielle de chercher à comprendre la pensée interne qui meut l'humanité et d'où naît la connaissance ${ }^{4}$, "

Agrégé de lettres en 1884, il enseignera aux lycées de Tours et de Douai, puis aux lycées Buffon, Louis-le-Grand et Henri-IV entre 1896 et 1925. Sa thèse de doctorat ès lettres, soutenue en 1898, porte sur L'Avenir de la philosophie. Esquisse d'une synthèse des connaissances fondées sur l'histoire; on y lit déjà un chapitre final sur «Le Règne de la synthèse » dont le lyrisme messianique est étonnant: «La Synthèse doit absorber toutes les énergies, dériver et raviver l'ancien esprit d'aventure. Se lancer à la conquête de tout l'inconnu ; contrôler l'hypothèse de l'Unité ; réaliser enfin les fins unifiantes de l'Être: n'est-ce pas une aventure assez passionnante ${ }^{5}$ ?»

En 1900, il fonde la Revue de synthèse historique; «notre cheval de Troie, dira Lucien Febvre, et par quoi s'insinuèrent dans la place tant de nouveautés ennemies et troublantes ». Elle avait simplement pour but de « rapprocher les diverses études historiques, de les éclairer l'une par l'autre, d'orienter la recherche et d'unifier les résultats ». Afin d'affirmer sa différence, en premier lieu avec la Revue historique de Gabriel Monod, fondée en 1876 , elle se propose de :

« Dresser, pour toutes les divisions de l'histoire, un état du travail fait et à faire [...]. Elle neutralisera les effets fâcheux d'une analyse et d'une spécialisation d'ailleurs nécessaires. Elle rejettera ce qui est vague ou arbitraire, mais elle s'appropriera ce qui est scientifique dans les recherches de l'anthropologie, de l'ethnologie et de la sociologie. En même temps qu'elle élargira, elle cherchera à approfondir et à préciser la conception de l'histoire. Elle amènera à collaborer non seulement les diverses équipes d'historiens, mais avec les historiens, les philosophes. Elle sera synthétique dans toute la force du $\operatorname{mot}^{6}$."

4. Henri BERR, En marge de l'histoire universelle, Paris, La Renaissance du livre, 1934, p. XII.

5. H. BERR, L'Avenir de la philosophie. Esquisse d'une synthèse des connaissances fondée sur l'histoire, Paris, Hachette et Cie, 1899, t. I, p. 510-511.

6. Prospectus de mai 1900 destiné à présenter la revue. 
Il prévoit quatre rubriques : article de fond, revue générale, notes et discussions, bibliographie. L'originalité des « revues générales » saute aux yeux, car elles parcourent «successivement tout le domaine de l'érudition, l'histoire entière de la pensée et de l'action humaine [...] sans rien négliger de ce qui concerne l'humanité agissante et pensante». Il précise :

«On voudrait surtout avoir et on compte obtenir une série d'articles sur la méthode des diverses sciences historiques. Faire ressortir ce qu'il y a de propre et ce qu'il y a de commun à l'histoire politique, à l'histoire économique, à l'histoire des religions, à celles de la philosophie, des sciences, de la littérature et des arts; recueillir les résultats de l'expérience, les réflexions d'esprits distingués qui se sont appliqués avec succès à telle ou telle partie de l'histoire; amener les philosophes à préciser une section importante de la logique des sciences qui, même dans les meilleurs traités, est encore vague et imparfaite : cela n'est peut-être pas son utilite ${ }^{7}$. »

«On entrait, se souvient Lucien Febvre, et dans un petit cabinet assez étroit, maussade et noir, on trouvait, derrière un bureau, un homme jeune, svelte, d'une mise sobre mais élégante, avec une grande barbe si foncée qu'elle a bien de la peine, aujourd'hui, à se mettre au gris. Beaucoup de visites toujours, dans le petit cabinet. Des jeunes et des anciens [...].On se frayait un passage au milieu des chaises. Et si petit jeune homme, si mince débutant que l'on fût, on connaissait votre accueil. Une parfaite bonne grâce, certes; une parfaite cordialité bien plus : un élan. Cet élan de l'esprit vers l'esprit, qui se sent tout de suite; cet appétit, ce fraternel respect de l'homme qui cherche pour l'homme qui vient chercher... Comme on vous en savait gré, mon cher ami, de cet accueil! Comme on sortait de chez vous réconforté, soulagé, plus riche de confiance dans la vie, plus décidé à la rendre utile et belle, à la féconder par son labeur ${ }^{8}$."

En 1911, dans La Synthèse en histoire, il explique : «Pour réagir contré les excès de l'analyse et de la spécialisation, pour approfondir les problèmes théoriques de l'histoire, pour mettre en rapports réguliers les historiens et les philosophes, j'ai fondé la Revue de synthèse historique ${ }^{9} \gg$; puis, dans la présentation de l'anthologie des Avant-Propos des volumes de «L'Évolution de l'humanité » publiée en 1934 :

7. «Sur notre programme », Revue de synthèse historique, I, 1, 1900, p. 1-2.

8. Lucien FebVRE, «Hommage à Henri Berr pour ses quatre-vingts ans », Revue de synthèse, III ${ }^{e}$ série, 35, juil.-sept. 1964, p. 8-12, en particulier p. 9. Les locaux de la revue étaient alors 12, rue Sainte-Anne.

9. La Synthèse en histoire. Son rapport avec la synthèse générale, Paris, 1911, 2e éd. A. Michel («L'Évolution de l'humanité »), 1953, p. XIII. 
«En 1900, la Revue de synthèse historique troublait beaucoup d'esprits en annonçant qu'elle embrassait dans son programme toutes les activités humaines, sans exception : aujourd'hui, de nombreuses Revues et les Congrès quinquennaux d'histoire s'ouvrent largement à la diversité de ce qu'on nomme "les sciences historiques". Progrès sensible; formule regrettable. C'est une "discipline" que cultive le spécialiste, non une science - une discipline qui apporte à la science sa contribution. Il n'y a pas des "sciences historiques", il n'y a qu'une "science de l'histoire", on ne l'affirmera jamais trop ${ }^{10}$. »

La deuxième grande entreprise fut la conception et la direction d'une célébrissime collection «L'Évolution de l'humanité ». Dès 1910, il pense à une histoire universelle et en conçoit le plan en 1912; le premier tome devait paraître durant l'automne 1914. Prévue en cent volumes, elle naît en 1920 et sera publiée chez Mignot et Tallandier, puis à La Renaissance du livre et enfin, à partir de 1937, chez Albin Michel où elle se trouve encore aujourd'hui. La correspondance, largement inédite ${ }^{11}$, le montre en train de choisir les collaborateurs, discuter plans et sujets, consulter, se raviser puis décider. Le plan global ne manquait pas d'audace car Berr concevait la collection comme un tout : « Cette "synthèse collective" devait être autre chose qu'une collection de monographies excellentes. » Ses Avant-Propos devaient en souligner la logique interne : «La synthèse, ici, ne consiste pas seulement, ne consiste pas surtout, à réunir en une seule histoire toutes les histoires spéciales, - politique, économique, religieuse, intellectuelle $^{12}$. » Grâce à la Revue de synthèse, cela pouvait sembler acquis... Dans son «Introduction générale, l'évolution de l'humanité - synthèse collective », il rassemble ses arguments :

«L'Allemagne, depuis une vingtaine d'années, a donné le spectacle d'une floraison de l'Histoire universelle - sous le nom de Weltgeschichte. Dans ce pays de l'érudition, mais aussi des synthèses aventureuses, où l'on sait mal tenir l'équilibre entre la micrographie et la métaphysique, l'ardent labeur des historiens et la préoccupation mondiale ont abouti à la publication d'œuvres nombreuses, inégales en importance et en intérêt, qui ont cherché à satisfaire et qui ont excité en même temps l'appétit d'histoire universelle. [...] On pouvait se demander pourquoi la France, à son heure, n'emploierait

10. Op. cit. supra n. 4 , p. VI.

11. Outre le dépôt de l'IMEC en cours d'exploitation, des lettres de et à Berr se trouvent au département des manuscrits de la BNF aux Archives nationales, dans celles de l'Unesco et du Collège de France et, sans doute, dans de nombreux fonds étrangers. Un catalogue systématique s'impose ! On attend avec impatience la publication de plus de 300 lettres entre Febvre et Berr.

12. Op. cit. supra n. 4 , p. VI. 
pas les ressources en hommes de science dont elle dispose, elle aussi, n'utiliserait pas surtout son génie propre, ce besoin de clair et profond savoir, pour une vaste entreprise qui embrasserait l'Humanité, depuis ses origines, et la Terre dans toute son étendue ${ }^{13}$.»

Commentant, en 1920 , ce texte qui devait paraître en 1914 , il ajoute :

« Et nous voulions donner un exemple de courage intellectuel. Plus que jamais notre initiative semblera opportune. Il faut que, dans ce domaine aussi, la vitalité de la France se manifeste. Il faut que, dans ce domaine aussi, notre supériorité sur l'Allemagne éclate. Malgré ses mérites, la science allemande s'est compromise souvent par sa subordination à des fins égoïstes. Il y a eu des "vérités allemandes". La science française n'est pas nationale : elle est l'apport d'une nation au trésor intellectuel de l'humanité. Elle se voue à la recherche de la vérité intemporelle et sans patrie ${ }^{14}$."

Après les diverses modifications du plan général, il semble s'être arrêté à quatre sections : Introduction (préhistoire, protohistoire), Antiquité, origines du christianisme et Moyen Âge, le monde moderne, et enfin vers le temps présent.

« Notre programme est immense, et notre ambition paraîtra téméraire à certains. Mais il faut oser. On parle beaucoup, depuis quelque temps, de "renaissance française" : il est visible que le goût de l'action, que la confiance dans les énergies spontanées de la vie sont ranimées chez nous. Cette disposition aurait un côté inquiétant si, comme quelques-uns l'annoncent, elle devait être anti-intellectualiste. Il convient que ce besoin d'agir et ce réveil d'énergie se manifestent par le courage intellectuel. La vie s'épanouit dans la connaissance. Et une science historique virilement comprise - conscience réfléchie de l'humanité - est nécessaire pour diriger les puissances tumultueuses de l'instinct ${ }^{15}$.

Il concevait son travail comme celui d'un véritable laboratoire :

« Notre cuvre, tout en rendant les services d'une Encyclopédie, sera autre chose, on le voit, qu'une Encyclopédie. Si un peu de science stérilise l'histoire, beaucoup de science doit la vivifier. La préoccupation des causes générales, éternelles, qui peut rehausser la recherche la plus humble, donnera ici

13. Imprimé dans le vol. I de la collection, Edmond PERRIER, La Terre avant l'histoire, Paris, La Renaissance du livre, 1920, p. IV.

14. Ibid., p. XXV.

15. Ibid., p. XXIV. 


\begin{abstract}
à la Synthèse non seulement toute sa dignité, mais son plein intérêt et comme un attrait dramatique. Il s'agit en somme, de refaire, derrière l'humanité, le chemin qu'elle a suivi; il s'agit de le refaire, - ce chemin que l'instinct aveugle, que des puissances obscures, que des circonstances multiples lui ont imposé, - en comprenant pourquoi elle l'a parcouru. Sur la route des temps, parmi les efforts, les ambitions, les luttes, les destins divers des groupes, malgré les piétinements, les détours et les reculs, l'humanité monte. En montant, elle embrasse de plus haut l'horizon; elle tâche, avec les historiens, à se situer dans l'espace et la durée, à prendre conscience d'elle-même, à savoir pour pouvoir mieux. Ainsi une entreprise comme celle-ci est un acte. Et si l'historien a le devoir, comme savant, de recueillir les faits et de rechercher les causes objectivement, impassiblement, il a le droit, comme homme, de se passionner pour son travail et de l'animer d'une flamme intérieure ${ }^{16}$. »
\end{abstract}

À sa mort, en 1954, 65 volumes sont parus, souvent réédités, largement traduits; ils sont toujours aujourd'hui une référence obligée et incontournable des étudiants et de leurs professeurs.

La troisième et dernière entreprise de Berr fut la création de la Fondation "Pour la Science » - Centre international de synthèse, l'année de sa retraite, en 1925, qui s'installera, grâce à Paul Doumer, en 1927, dans l'Hôtel de Nevers, au 12 de la rue Colbert, Paris, IIe, où elle est toujours. La lecture des quelques Bulletins du Centre international de synthèse permet de sentir un peu de sa prodigieuse activité.

Entre la science et le public, entre l'économie et l'art, entre l'université et la politique, le CIS tissa un filet de connaissances fascinant à explorer. Le salon de son épouse Cécile (1876-1963), née Halphen, élargira encore le cercle de ses relations. Berr est en relation épistolaire avec toute l'Europe, surtout l'Allemagne et l'Italie, et les États-Unis. Au conseil d'administration, on trouve des grands commis de l'État ${ }^{17}$, des politiques, des banquiers, mais aussi Einstein, Frazer, Rutherford, Herriot et Luchaire. En 1927, il écrit :

«L'objet du Centre international de synthèse est de travailler à l'unification des sciences historiques, à l'unification des sciences de la nature, à l'unification enfin de ces deux ordres de connaissances; pour cela, de mettre en lumière les grands résultats du travail scientifique, de préciser les problèmes d'interscience, d'en ménager et d'en hâter la solution ${ }^{18}$. "

16. Ibid., p. XIX-XX.

17. Je remercie Jean-Noël Jeanneney d'avoir mis à ma disposition la correspondance de son grand-père avec Henri Berr et d'en avoir autorisé la copie, désormais accessible à l'IMEC.

18. Bulletin du Centre international de synthèse, 4, déc. 1927, p. 41, Revue de synthèse historique, 130-132, déc. 1927. 
Un an plus tard, il précise : «Le CIS n'est pas une société savante, ni un nouvel institut de l'enseignement supérieur, mais un instrument de réactivation de la réflexion régulatrice, un terrain d'exploration systématique et rationnelle de la logique des sciences en vue de leur intégration réciproque ${ }^{19}$.» Il affirmera souvent l'unicité du Centre :

« Je vous rappelle, en effet, que notre fondation a un caractère original, non pas seulement par son objet propre, qui est de compléter, en les couronnant, les diverses institutions scientifiques, mais par son mode d'activité : s'il n'est pas conçu comme un établissement d'enseignement supérieur, il ne ressemble en rien, d'autre part, à une société savante. Un effort continu y soutient, non pas seulement les débats où l'on s'instruit mutuellement, mais des entreprises de longue haleine, qui sont menées avec autant de diligence qu'il est possible $^{20}$.»

Le Centre, dont le secrétaire général était André Tolédano, comportait au départ trois sections : synthèse historique, sous la direction d'Henri Berr et Lucien Febvre, sciences de la nature, sous la direction d'Abel Rey puis de Paul Langevin, et enfin synthèse générale. Très vite, un autre domaine lui apparaîtra capital :

«L'histoire des sciences, jusqu'à présent, a été trop peu cultivée et elle l'a été d'une façon trop décousue et tâtonnante, ou encore trop purement technique. Or elle se trouve établir une étroite liaison entre les sciences de la nature et celles de l'humanité. Vue sous un certain angle, elle est l'inventaire méthodique des progrès lentement accomplis dans l'interprétation et la conquête de la nature; sous un autre aspect, elle étudie la pensée scientifique, - un élément de la vie humaine, - mêlée aux autres éléments, et qui agit puissamment sur eux. Pour la synthèse des connaissances, qui est notre objet propre; pour la synthèse de la spéculation et de l'action, - qui est la fin der-, nière des efforts même les plus désintéressés provisoirement, — rien n'a plus de prix que l'histoire des sciences ${ }^{21}$. »

Elle aura donc elle aussi sa section.

Ce Centre sera la base logistique du Vocabulaire historique et des Semaines de synthèse. En 1931, la Revue de synthèse historique devient, sous le nom de Revue de synthèse, l'organe du Centre. Avec la naissance des Annales (1929), fondée par deux collaborateurs éminents et réguliers,

19. Conseil d'administration du 25 mai 1928, in G. Gemelli, op. cit. supra n. 1, p. 250.

20. Bulletin du Centre international de synthèse, 6, déc. 1928, p. 49, Revue de synthèse historique, 136-138, déc. 1928.

21. lbid., p. 50. 
et l'Encyclopédie française (après 1932), les liens vont un peu se distendre mais pas le travail commun, et la dette sera souvent réaffirmée. Saluant les qualités d'organisateur de Febvre, il mentionnera plus tard les Annales créées « pour éclairer un aspect de la vie des sociétés, resté trop longtemps dans l'ombre et sur lequel le marxisme avait appelé l'attention ${ }^{22}$ ».

De marginal qu'il était, né littéraire, philosophe et historien, deux fois candidat malheureux au Collège de France (1903, 1912), il est entre les deux guerres au centre d'une galaxie intellectuelle qui semble sans limites. Il rend compte de tout, reçoit tout le monde, fréquente les réunions internationales et préside la section «méthode historique et théorie de l'histoire » au congrès de Varsovie en 1933. L'année suivante, il affirme crânement: «La science aura le dernier mot. » Deux ans avant sa mort, il se souvient:

«Nous avons, au Centre de synthèse, essayé, dans nos "Semaines", de faire éclater cette conclusion que tout est histoire puisque tout est évolution; que de la matière à l'esprit il y a eu continuité et progrès; que la réflexion philosophique a posé des problèmes dont les solutions, peu à peu, sont données par la science ; et ainsi, c'est en vue de l'histoire totale, que l'histoire humaine, scientifiquement comprise, doit éclairer les philosophes - bien loin de leur demander des lumières. À plus forte raison doit-elle, par sa propre systématisation, introduire un ordre foncier dans toutes les formes de contribution à l'étude de l'humanité ${ }^{23}$.»

Une vingtaine de volumes des actes des Semaines de synthèse, où se côtoie le gratin de l'intelligence du temps, offre un corpus qu'on lit encore aujourd'hui avec profit et, pourquoi ne pas le dire, pas mal d'admiration ! À propos de la première Semaine de synthèse, en mai 1929, consacrée à la civilisation et à l'évolution biologique, il indique clairement ses buts :

« Nous commencerons ainsi à réaliser une partie importante de notre programme originel, et qui, peut-être, est assez neuve. Nous ne donnerons pas ici d'enseignement. Nous ne donnerons pas de "conférences" selon la formule banale. Nous ne voulons ni des élèves, ni un public d'amateurs. Nous ne méprisons pas les congrès, mais nous estimons qu'on peut concevoir des réunions, même internationales, où l'on ne parcoure pas, soit la science, soit une partie de la science à vol d'oiseau; où l'effort ne s'éparpille pas sur une foule de sujets, ne s'amenuise pas en questions de détail ${ }^{24}$. »

22. La Synthèse en histoire, op. cit. supra n. 9, p. 289.

23. Ibid., p. $\mathrm{x}$.

24. Bulletin du Centre international de synthèse, 7, juin 1929, p. 45, Revue de synthèse historique, 139-141, juin 1929. L'inauguration officielle du Centre eut lieu le 22 mai 1929 à 16 heures. 
Il faut évoquer aussi cette grande entreprise que fut le Vocabulaire historique, dont on espère une prochaine publication d'extraits. Le Bulletin en offre d'excellents exemples. En 1936, il lance la Revue d'histoire des sciences, la seconde revue du Centre international de synthèse.

On ne sait pas encore grand-chose de sa vie pendant la Seconde Guerre mondiale, alors que la collection «L'Évolution de l'humanité » s'enrichit du célèbre Problème de l'incroyance de Lucien Febvre et qu'il publie sous son nom le roman Hymne à la vie, mais on sait que certains de ses livres figurent sur la liste Otto, que les Allemands interdisent et pilonnent les brochures de sa collection Descartes «Pour la vérité », et qu'il refuse de porter l'étoile jaune. Le 2 février 1943, Lucien Febvre réunit quelques amis pour son anniversaire et lui lut les paroles reproduites dans un Hommage à Henri Berr pour ses quatre-vingts ans, imprimé clandestinement. Ce dernier reçoit le 11 février une superbe lettre de Marc Bloch, deçu de ne pas être présent :

«J'aurais voulu pouvoir être là, afin de vous dire ce que tant d'entre nous sentent et que je sens, je crois, avec une force particuliere : tout ce que nous devons comme historiens qui cherchent a faire de la vraie histoire, à votre largeur de vues, à votre pénétration, à vos audaces; tout ce que je dois personnellement à votre amitié; - aussi bien comment séparer? votre action n'a été si féconde que parce qu'elle a été, avant tout, cordialement et généreusement humaine [...]. Ma pensée, du moins, va vers vous, avec une respectueuse amitié, vers le bel exemple de courage que vous nous avez donné en ces jours tragiques; vers vos deux demeures ${ }^{25}$ naguère si accueillantes et qui, j'en suis sûr, le redeviendront bientôt. Car voici qu'à l'horizon pointe une aube encore timide, mais qui sans cesse devient plus lumineuse. Nous aurons encore nos épreuves. Elles seront l'annonce du matin, du retour et du revoir $^{26} \ldots$ »

En 1952, sa collaboratrice Suzanne Delorme évoque l'activité de Berr:

« $\mathrm{A} 89$ ans, cet homme étonnant monte encore, malgré une fracture récente du col du fémur, les cent marches du Centre, pour recevoir disciples et amis, dans le salon de Madame de Lambert ou dans le petit bureau de l'Abbé Bar-

25. Il s'agit du Centre international de synthèse et de son appartement du 2, rue Villebois-Mareuil, à Paris, XVIIe.

26. Marc Bloch, Écrire La Société féodale. Lettres à Henri Berr, 1942-1943, p. 113-114. Cf. aussi Natalie Zemon Davis, «Rabelais among the Censors (1940s-1950s) », Representations, 32, 1990, p. 1-32, et les remarques de Peter SchöTtLER, « Marc Bloch, Lucien Febvre face à l'Allemagne nazie », Genèses, 21, 1995, p. 75-95. 
thélemy, avec la même aménité qu'autrefois, discutant avec la même vivacité d'esprit, la même précision des termes, la même richesse de pensée, la même vigueur de raisonnement qui l'ont toujours caractérisé. À 89 ans, il travaille du matin au soir, écrit, parle, reçoit comme à 50, sans se laisser troubler par les événements extérieurs, mais non sans les juger ${ }^{27}$.»

Lorsqu'il disparaît en 1954, il laisse un héritage qu'une poignée de fidèles réussiront à faire fructifier contre vents et marées, grâce à Suzanne Delorme, Paul Chalus et Jacques Roger. Ce dernier, avec Jean-Claude Perrot et Ernest Coumet, a redonné un nouvel élan au Centre, à sa revue et à sa collection. C'est à lui que nous avons pensé en préparant ce colloque. Et à l'heure où nous en préparons les actes disparait de manière brutale Bernard Lepetit, codirecteur de la collection pour laquelle il n'avait ménagé ni son temps - qui était compté - ni sa science qui était grande.

Derrière le «tout comprendre et tout unir» se profile une double exigence du cœur et de l'esprit, l'impératif d'intelligence du monde et de la communication des savoirs. La synthèse fut d'abord un dialogue entre les hommes, les disciplines, les pays et les mondes. Loin de n'être qu'une préhistoire d'une revue plus connue, elle reste le témoignage d'une volonté d'innovation, d'audace intellectuelle qui anime tous les chercheurs véritables. Il est frappant que Berr n'a cessé d'affirmer la même chose, en 1900, avec la Revue de synthèse historique, en 1911, avec La Synthèse en histoire, depuis 1920, avec «L'Évolution de 1'humanité », puis, en 1925, avec la fondation du Centre international de synthèse. Pourtant, il semble ne pas avoir été compris; un an avant sa mort, il avoue: "Notre conception de la Synthèse scientifique n'a été [...] ni pleinement appliquée, ni même sérieusement discutée. Il semble que de cette "Synthèse en Histoire" on ait apprécié le détail et négligé l'essentiel ${ }^{28}$. » Mais derrière l'action, il y a une réflexion et dans le cas de Berr une grande pensée, finalement assez proche de Hegel mais sans la téléologie d'un système, car c'est la synthèse historique qui devait remplacer la philosophie de l'histoire :

«Et pourtant, si nous allons jusqu'au bout de notre pensée, nous dirons que l'histoire, telle que nous la comprenons, se relie à la synthèse générale, est à la base de la réflexion philosophique; qu'elle doit devenir, un jour, l'âme même de l'enseignement; qu'elle peut donner à tant d'êtres, à tant de jeunes surtout, en désarroi intellectuel, la joie d'embrasser le temps, de comprendre

27. Suzanne DELoRME, «Henri Berr », Osiris, X, 1952, p. 8.

28. La Synthèse en histoire, op. cit. supra n. 9, p. 289. 
la vie, de se dépasser, pour ainsi dire, en situant l'individu dans l'Humanité, et l'Humanité elle-même dans la totalité du $\operatorname{Ré}^{29}{ }^{29}$. 》

Il nous est agréable de remercier les nombreuses compétences qui assurèrent la réussite de ce colloque : nos hôtes de l'IMEC qui abrite désormais les papiers de Berr, tous les participants, ainsi que le CNRS, le CIS et la Ville de Paris. Les collaborateurs du Centre, dont Emest Coumet, n'ont pas ménagé leur peine et leurs conseils, et surtout Agnès Biard qui en fut comme d'habitude la cheville ouvrière. Grâce à leurs efforts et à leurs recherches, ce volume apporte une contribution considérable à son sujet. Il ouvre aussi quelques nouveaux chantiers que le Centre international de synthèse devra promouvoir et mener. Depuis tant d'années, et au mépris de toute logique commerciale, les éditions Albin Michel aident de manière décisive notre travail : à Francis Esménard, le PDG, une fois au moins, publiquement, va notre gratitude.

29. Ibid., p. 308. 


\section{BIBLIOGRAPHE}

Lucien Febvre, «De la Revue de synthèse aux Annales, Henri Berr, ou un demi-siècle au service de l'histoire ", Annales ESC, 1952, p. 289-292.

Suzanne Delorme, «Henri Berr», Osiris, X, 1952, p. 5-9.

Henri Berr (1863-1954), Paris, 1955.

Suzanne DELORME, «La naissance d' Isis. Premiers rapports entre Henri Berr et George Sarton ", Actes du XI Congrès international d'histoire des sciences, 24-31 août 1965, Wroclaw-Varsovie-Cracovie, Ossolineum, maison d'édition de l'Académie polonaise des sciences, 1967, II, p. 223-232.

Barbara ARCangeli et Margherita Platania (sous la dir. de), Metodo storico e scienze sociali : la RSH (1900-1930), Rome, Bulzoni, 1981.

Martin SIEGEL, "Henri Berr et la Revue de synthèse historique", in Charles-Olivier CARBONELL et Georges LIVET (éd.), Au berceau des Annales. Le milieu strasbourgeois. L'histoire de France au début du XXe siècle, Toulouse, Presses de l'IEP de Toulouse, 1983, p. 205-218.

Martin FugLER, Analyse ou synthèse? La Revue de synthèse historique et l'histoire (19001910), sous la dir. de François HARTOG et Pierre AYÇOBERRY, mémoire de maîtrise dactylo., université de Strasbourg, 1985.

Giuliana GEMELLI, « Communauté intellectuelle et stratégies institutionnelles. Henri Berr et la fondation du Centre international de synthèse », Revue de synthsèse, CVIII, 2, avr.-juin 1987, p. 225-259.

Marc BLOCH, Écrire La Société féodale. Lettres à Henri Berr, 1924-1943, correspondance établie et présentée par Jacqueline Pluet-Despatin, préface de Bronislaw Geremek, Paris, Institut Mémoires de l'édition contemporaine, 1992.

Jacqueline PluET-DesPatiN, «Lucien Febvre et la Revue de synthèse », La Revue des revues, 14, 1992, p. 3-7.

On trouvera bien entendu de multiples renseignements dans les travaux consacrés aux Annales et à leurs fondateurs:

François Dosse, L'Histoire en miettes. Des «Annales» à la «nouvelle histoire », Paris, La Découverte, 1987.

Hartmut ATSMA et André BURGUière (éd.), Marc Bloch aujourd'hui. Histoire comparée et sciences sociales, Paris, EHESS, 1990.

Bryce et Mary LYON, The Birth of Annales History: The Letters of Lucien Febvre and Marc Bloch to Henri Pirenne, 1921-1935, Bruxelles, 1991.

Marc Bloch et Lucien Febvre. Correspondance, t. I : La Naissance des Annales, Bertrand Múller (éd.), Paris, Fayard, 1994.

Les Mots de l'histoire. Le "vocabulaire historique » du Centre national de synthèse, Margherita Platania (éd.), Naples, Bibliopolis, 1996. 